\title{
Intraocular gentamicin as intraoperative prophylaxis in South India eye camps
}

\author{
GHOLAM A. PEYMAN, M. LATHEEF SATHAR, AND DONALD R. MAY \\ From the Department of Ophthalmology, University of Illinois Eye and Ear Infirmary, Chicago, and \\ the Department of Ophthalmology, Government Erskine Hospital, Madurai, South India
}

SUMmARY The incidence of bacterial endophthalmitis has been $3.6 \%$ after cataract extraction in the eye camps of South India from 1961-75. During this time 50791 cases were operated using systemic and topical chloramphenicol prophylaxis.

This study evaluated the prophylactic intracameral injection of $50 \mu \mathrm{g}$ of gentamicin in eye camp cataract extractions. Only 6 of 1626 patients treated with intracameral gentamicin at operation developed bacterial endophthalmitis, an incidence of $0.37 \%$.

While the incidence of endophthalmitis in the welldeveloped countries has been reduced to acceptable levels of 0.1 to $0.2 \%$ (Allen and Mangiaracine, 1964, 1973), the disorder is still one of the major complications after cataract extraction in the developing countries. This has been the case especially in the eye camps in South India, where large numbers of patients (up to 500 per day) are operated on. These eye camps serve the rural masses who cannot afford the expense of coming to a hospital which may be as far as 80 miles $(130 \mathrm{~km})$ from their village.

In the past, before the introduction of intraocular gentamicin as prophylaxis against endophthalmitis, each patient was given $250 \mathrm{mg}$ of chloramphenicol by mouth 8 hours before surgery. Chloramphenicol ophthalmic drops were instilled in the eye 1 hour before surgery. Postoperatively the patients were given chloramphenicol $250 \mathrm{mg}$ by mouth every 8 hours and $1 \%$ chloramphenicol eye drops were instilled at daily dressing changes.

Additional preoperative medication included $10 \%$ phenylephrine and $1 \%$ atropine eye drops 1 hour before surgery. In addition to the $250 \mathrm{mg}$ of chloramphenicol $25 \mathrm{mg}$ of chlorpromazine was given orally 1 hour preoperatively. Facial and retrobulbar injections of $1 \%$ lignocaine solution were given immediately preoperatively.

The surgeon's and nurse's hands were scrubbed and rinsed with an ether-soap mixture. Operating staff put on sterile gowns, which were used throughout the operating day. Gloves were not worn, all surgery being done with bare hands. The hands

Address for reprints: Donald R. May, MD, 1855 W. Taylor Street, Chicago, Illinois 60612, USA were rinsed between cases with sterile water and occasionally washed again with the ether-soap mixture. Surgery was done almost entirely by Graefe knife section, and the wound was closed with 1 to 3 interrupted corneoscleral sutures. Instruments were placed in boiling water for a few minutes between cases.

The patients were seen postoperatively on a daily basis by the camp ophthalmologist. Atropine 1\% drops and chloramphenicol $1 \%$ drops were applied at each dressing change. The patients were discharged on the seventh postoperative day with instructions to return to the area hospital for at least 1 postoperative follow-up examination. On discharge from the camp each patient was given a pair of cataract glasses equal to their retinoscopic spherical equivalent.

On this regimen the endophthalmitis rate was $3.6 \%$. Between 1961 and 1975 a total of 50791 cases were operated upon in the above manner, and 1825 developed bacterial endophthalmitis (Sathar, 1976). The patients with endophthalmitis were treated with intramuscular tetracycline twice daily and oral prednisolone daily. Intraocular injections of antibiotic solutions, including chloramphenicol and gentamicin, were also initiated during the past 3 years. In our study, when intracameral injection was used, $0.2 \mathrm{ml}$ of a saline solution containing $50 \mu \mathrm{g}$ of gentamicin was injected into the anterior chamber of each eye immediately after placement of the corneoscleral sutures. As in the previous eye camps, the surgeons were attending and house staff ophthalmologists from the Government Erskine Hospital, Madurai. No changes were made 
Table 1 Incidence of endophthalmitis and other complications among the operated cases

\begin{tabular}{|c|c|c|c|c|c|c|c|}
\hline Camp no. & Girous & $\begin{array}{l}\text { No. of } \\
\text { casc's }\end{array}$ & $\begin{array}{l}\text { Anterior chamber } \\
\text { shallow }\end{array}$ & $\begin{array}{l}\text { Cortex retaincel in } \\
\text { anterior chamber }\end{array}$ & Endophithalmitis & Hyphe'ma & $\begin{array}{l}\text { Iris } \\
\text { prolapse }\end{array}$ \\
\hline 1 & 1 & 219 & 6 & 11 & 6 & 2 & - \\
\hline 2 & 2 & 369 & 16 & 4 & 5 & 3 & 7 \\
\hline 3 & 3 & 146 & 5 & 8 & 1 & 3 & $\ldots$ \\
\hline 4 & 3 & 195 & 19 & 12 & 1 & 2 & $\ldots$ \\
\hline 5 & 3 & 302 & 6 & 9 & 2 & 5 & - \\
\hline 6 & 3 & 260 & 6 & 14 & 2 & 8 & 3 \\
\hline 7 & 3 & 168 & 5 & 1 & & 4 & 2 \\
\hline 8 & 3 & 367 & 4 & 6 & - & 3 & 2 \\
\hline Total & & 2026 & 67 & 65 & 17 & 30 & 14 \\
\hline
\end{tabular}

in the original procedure or in the preoperative selection and preparation of the patients or in their postoperative care. The camps in our study were sequentially divided into 3 groups:

Group I: No prophylactic intracameral gentamicin was given. Oral and topical chloramphenicol was used for all patients as described in the original protocol.

Group 2: Prophylactic intracameral gentamicin was used for the female patients only. No chloramphenicol was used for the female patients. Oral and topical chloramphenicol was given to all male patients as described.

Group 3: Prophylactic intracameral gentamicin was used for all patients in this group. No chloramphenicol or any other antibiotic was given to any of these patients.

\section{Results}

Group 1: In camp 1 a total of 219 cataract extractions were performed. Six eyes developed postoperative endophthalmitis, an incidence of $2.9 \%$ in this camp.

Group 2: In the second camp all the female patients received intracameral gentamicin, and the male patients were treated as in Group 1. A total of 188 female patients had cataract extractions, with no cases of endophthalmitis. Five of the 181 male patients having cataract extractions developed endophthalmitis, an incidence of $2 \cdot 8 \%$.

Group 3: In camps 3 to 8 all patients received intracameral gentamicin. Among 1438 patients in this group there were 6 cases of postoperative endophthalmitis, an incidence of only $0.42 \%$.

The combined incidence of postoperative endophthalmitis in the patients in group 3 and those treated with intraocular gentamicin in group 2 was $0.37 \%$.

Table 1 summarises the postoperative complications in each camp including the cases of endophthalmitis. 11 cases of endophthalmitis were diagnosed in groups 2 and 3 . Aspiration of $0.1 \mathrm{ml}$ of vitreous was performed in each of these cases, and cultures were done using cooked meat and chocolate agar media. Each of the 11 cases was culture positive as listed in Table 2.

All the cultured organisms were sensitive to gentamicin. Each case of endophthalmitis was treated with a combination of gentamicin and dexamethasone. A total of $400 \mu \mathrm{g}$ of gentamicin and $360 \mu \mathrm{g}$ of dexamethasone was given by a single intravitreal injection according to our technique (May et al., 1974; Peyman et al., 1974a; Peyman et al., 1974b). The ocular media cleared in 9 of the 11 cases after this single treatment. The other 2 eyes did not clear completely, and eventually progressed to phthisis bulbi.

\section{Discussion}

The rate of endophthalmitis in the South Indian eye camps has been $3.6 \%$ over the past 14 years. This is comparable to the $2.9 \%$ incidence of endophthalmitis in those patients in our series not treated with intracameral gentamicin. This high incidence is attributable to the inability to achieve asepsis in the temporary operating rooms prepared in the village eye camps. Even with the best of efforts flies are present and sterile technique cannot be achieved.

Of 1626 patients receiving prophylactic intraocular gentamicin at operation only 6 developed bacterial endophthalmitis, an incidence of $0.37 \%$. This study shows that a substantial decrease in the incidence of endophthalmitis has been achieved with

Table 2 Results of vitreous aspirate culture

\begin{tabular}{lc}
\hline Organism cultured & No. of cases \\
\hline Staphylococcus albus & 3 \\
Coliform and S. aureus & 1 \\
S. aureus & 2 \\
Beta-haemolytic streptococcus & 1 \\
S. pyogenes & 1 \\
Pseudomonas aeruginosa & 3 \\
\hline Total & 11 \\
\hline
\end{tabular}


this method. We believe that the routine intraocular injection of small quantities of gentamicin, i.e., $50 \mathrm{\mu g}$, could greatly reduce the incidence of postoperative endophthalmitis in many parts of the world where surgical asepsis is a problem.

\section{References}

Allen, H. F., and Mangiaracine, A. B. (1964). Archives of Ophthalmology, 72, 454.
Allen, H. F., and Mangiaracine, A. B. (1973). Transactions of the American Academy of Ophthalmology and Otolaryngology, 77, 581.

May, D. R., Ericson, E. S., Peyman, G. A., and Axelrod, A. J. (1974). Archives of Ophthalmology, 91, 487.

Peyman, G. A., May, D. R., Ericson, E. S., and Apple, D. (1974a). Archives of Ophthalmology, 92, 42.

Peyman, G. A., Vastine, D. W., Crouch, E. R., and Herbst, R. W., Jr (1974b). Transactions of the American Academy of Ophthalmology and Otolaryngology, 78, 862.

Sathar, M. L. (1976). Personal communication. 